\title{
Access to Improved Sanitation and Nutritional Status among Preschool Children in Nouna District, Burkina Faso
}

Mamadou Bountogo, ${ }^{1}$ Mamadou Ouattara, ${ }^{1}$ Ali Sié, ${ }^{1}$ Guillaume Compaoré, ${ }^{1}$ Clarisse Dah, ${ }^{1}$ Valentin Boudo, ${ }^{1}$ Alphonse Zakane, ${ }^{1}$ Elodie Lebas, ${ }^{2}$ Jessica M. Brogdon, ${ }^{2}$ William W. Godwin, ${ }^{2}$ Ying Lin, ${ }^{2}$ Benjamin F. Arnold, ${ }^{2,3}$ and Catherine E. Oldenburg ${ }^{2,3,4 \star}$ for the Étude CHAT Group

${ }^{1}$ Centre de Recherche en Santé de Nouna, Nouna, Burkina Faso; ${ }^{2}$ Francis I Proctor Foundation, University of California, San Francisco, California; ${ }^{3}$ Department of Ophthalmology, University of California, San Francisco, California; ${ }^{4}$ Department of Epidemiology and Biostatistics, University of California, San Francisco, California

\begin{abstract}
Access to improved sanitation and hygiene may improve child nutritional status by reducing exposure to enteric pathogens. We evaluated this relationship as part of the Community Health with Azithromycin Trial, a communityrandomized trial of azithromycin versus placebo for the prevention of child mortality in rural Burkina Faso. Before the baseline study visit, a door-to-door household survey was conducted for all households in the study area. During the baseline study census, which occurred approximately 9 months after the household survey, a mid-upper arm circumference (MUAC) measurement was obtained from each child. We evaluated the relationship between household improved latrine use compared with unimproved latrines or open defecation and MUAC in children aged 6-59 months. Among 32,172 children with household survey data and MUAC measurements, 931 (2.9\%) had an MUAC less than $12.5 \mathrm{~cm}$ and were classified as having moderate acute malnutrition (MAM). The odds of MAM were higher in children living in households with an unimproved latrine than those with an improved latrine (adjusted odds ratio: 1.60; 95\% Cl: 1.11-2.31). Children in households with unimproved latrines and households that practiced open defection had approximate $0.15 \mathrm{~cm}$ reduced MUAC compared with those in households with an improved latrine. There was a small, but statistically significant, association between improved latrine and nutritional status in preschool children as measured by MUAC.
\end{abstract}

\section{INTRODUCTION}

Enteric and other infections are thought to lead to acute weight loss, which can lead to acute and/or chronic undernutrition. Access to clean water, sanitation, and hygiene (WASH) are hypothesized to reduce undernutrition by reducing fecal-oral transmission of enteric pathogens. ${ }^{1}$ Recent large-scale randomized controlled trials have failed to find evidence that improved access to WASH affects linear growth. ${ }^{2-4}$ By contrast, many observational studies have shown a significant association between WASH interventions and improved linear growth. ${ }^{1,5}$ These discrepancies may arise from unmeasured confounding in observational studies, challenges with behavior change in interventional studies, or failure of some elements of improved WASH to substantially reduce pathogen transmission and reduce chronic undernutrition over time. ${ }^{1,6}$

Infection has been hypothesized to cause acute malnutrition in addition to chronic undernutrition. Children with diarrhea who have acute malnutrition are at higher risk of mortality than their well-nourished peers. ${ }^{7}$ The relationship between infection and acute malnutrition is complex. For example, diarrhea can lead to impaired weight gain via reduced nutrient intake and malabsorption, and malnutrition can lead to increased susceptibility to or severity of diarrhea. ${ }^{8,9}$ Interventions that interrupt transmission of infection may also have an impact on nutritional status.

Mid-upper arm circumference (MUAC) is a measurement commonly used in community-based programs to screen for acute malnutrition. Mid-upper arm circumference is an indicator for wasting in children aged 6-59 months, and it has

\footnotetext{
${ }^{*}$ Address correspondence to Catherine E. Oldenburg, Francis I Proctor Foundation, University of California, San Francisco, 513 Parnassus Ave., Rm. S309 San Francisco, CA 94611. E-mail: catherine.oldenburg@ucsf.edu
}

been shown to better predict mortality than other anthropometric measurements such as weight-for-height Z-scores (WHZs) in population-based samples of children. ${ }^{10}$ Although MUAC identifies different subsets of children compared with the $\mathrm{WHZ},{ }^{11}$ MUAC is considerably easier to measure than $\mathrm{WHZ}$ as it does not require expensive equipment, ability to read measurements, or calculation of Z-scores. Mid-upper arm circumference is therefore well-suited to communitybased screening, and evaluation of determinants of nutritional status as measured by MUAC may be useful for generating evidence for which children may be at increased risk of acute malnutrition as identified by MUAC. Here, we evaluated the relationship between household WASH characteristics and acute malnutrition as defined by MUAC using data from a large population-based prospective study among preschool children in Burkina Faso.

\section{METHODS}

Study setting and methods. This study took place in Nouna district in northwestern Burkina Faso. The parent study for the present analysis encompasses the entire district, which includes both the Nouna Health and Demographic Surveillance Site (HDSS; which covers approximately one-third of the district) and all communities in the district not included in the HDSS. $^{12,13}$ In the present analysis, only communities outside the HDSS were included. Data arose from a pre-study mapping and survey and baseline census for a communityrandomized controlled trial of mass azithromycin distribution compared with placebo for prevention of child mortality. ${ }^{13,14}$ No azithromycin or placebo treatments occurred before study assessments. The study area is situated in the Sahel and is rural and agrarian. The malaria and malnutrition seasons overlap with the rainy season, with a higher prevalence of malaria and malnutrition from July through October. The household survey, which included water and sanitation 
assessments, occurred from December 2018 through April 2019, and the baseline study census, which included assessment of nutritional status, occurred from August 2019 through January 2020. The study was reviewed and approved by the Comité National d'Ethique pour la Recherche (National Ethics Committee of Burkina Faso) in Ouagadougou, Burkina Faso, and the Institutional Review Board the University of California, San Francisco, in the United States. Written informed consent was obtained from each head of households for participation in the household survey and census and from each child's caregiver for participation in the study.

Household survey. Before the start of the parent trial, a prestudy mapping exercise and household survey were undertaken in 228 villages in 10 communes of Nouna district, as previously described. ${ }^{14}$ Trained enumerators visited each structure in the communities and classified structures as inhabited or uninhabited. Each household was assigned a unique identifying number. The head of each household was interviewed in each inhabited structure and asked to list the number of males and females residing in the household, including each child younger than 5 years, and their dates of birth, gender, religion, and ethnicity. Heads of households then answered questions about the household's structure (such as number of rooms in the household) and assets, including household ownership of radios and mobile telephones. A household wealth index was constructed using a principal components analysis, combining responses for 20 different household assets, including radios, televisions, refrigerators, stoves, telephones, bicycles, and lamps.

Water and sanitation assessment. During the pre-study household survey, heads of households were interviewed about the type of toilet most commonly used by the household, which was categorized as improved latrines (e.g., with a slab), unimproved latrines (no slab), or no latrine/toilet (open defecation). They were then asked about where the household primarily gets its water for cooking and drinking, which was categorized as shallow well, drilled well (borehole), or a nonwell source in the dry season and separately in the rainy season. All WASH variables collected during the household survey are included in this report. Because of time and financial resources, additional WASH measurements were outside of the scope of the parent study.

Nutritional status assessment. During the trial's baseline census, MUAC measurements were obtained for all children who were present at the time of the study who aged between 6 and 59 months. For each child aged between 6 and 59 months, a single MUAC measurement was taken by measuring the midpoint of the child's left arm. Mid-upper arm circumference measurements were collected by 36 enumerators who had been trained by study investigators. A team of supervisors oversaw MUAC measurement collection and provided feedback by following a checklist of items while observing enumerators collecting data. Checklist items include the arm used by the enumerator, the position of the child and their arm during measurement collection, if they measured the midpoint of the arm correctly, and if the MUAC tape was placed correctly (e.g., not too tight or too loose). Additional MUAC measurements were not collected either by the enumerator or the supervisor. Only a single MUAC measurement was obtained from each child because of time and resource constraints in the study. Children with MUAC < $11.5 \mathrm{~cm}$ were referred to a nutritional program for severe acute malnutrition (SAM). Children with MUAC $<12.5 \mathrm{~cm}$ were considered to have moderate acute malnutrition (MAM). Midupper arm circumference measurements were taken in a median of 9.4 months after the household survey (interquartile range [IQR]: 8.7-10.2 months). Mid-upper arm circumference measurements were not collected as part of the household survey.

Statistical methods. Descriptive characteristics were calculated overall and by whether or not the child had MAM, with medians and IQRs for continuous variables and proportions for categorical variables. We used logistic regression models to assess the association between 1) the type of latrine most commonly used by the household (categorized as improved latrine, unimproved latrine, or open defecation) and 2) the type of well (shallow or deep) used during the dry season and MAM as defined by the MUAC measurement, adjusted for the child's age and gender, the number of rooms in the household, and radio and mobile telephone ownership as proxies for socioeconomic status, with standard errors adjusted for clustering at the household level using a Huber-White sandwich estimator. We only modeled water source during the dry season because of lack of variability in water sources used between the dry and rainy seasons. We then used linear regression models adjusted for the same covariates to assess the relationship between the child's MUAC as a continuous variable and 1) latrine and 2) well type used by the household, with standard errors accounting for household-level clustering. A second set of models was run including the child's ethnicity and religion and the household wealth index as covariates. These were included in a separate model because of missing data in these variables. These models also included the child's age and gender and the number of room's in the household, with standard errors adjusting for clustering at the household level using a Huber-White sandwich estimator. Because of the low prevalence of SAM, we did not evaluate the relationship between latrine or well type and SAM. All analyses were conducted in Stata 15.1 (StataCorp, College Station, TX).

\section{RESULTS}

We linked 32,165 children aged 6-59 months in 17,311 households between the two surveys. Median MUAC was 14.5 (IQR: 13.9-15.4) cm; 931 (2.9\%) children had MAM; and $89(0.3 \%)$ children had SAM. Mean MUAC was 14.6 (SD: 1.2$)$ $\mathrm{cm}$. All, except one, MUAC measurements were in the 9-19.5 cm range. Approximately half of the children were female, but children with MAM were slightly more often female than male (Table 1). Children with MAM were younger than children without MAM (Table 1).

Children living in a household with an unimproved latrine had increased odds of MAM as determined by MUAC compared with those living in a household with an improved latrine (adjusted odds ratio: 1.60; 95\% Cl: 1.11-2.31; Table 2), but this relationship did not persist after adjusting for ethnicity, religion, and household wealth index. The absolute difference in prevalence of MAM was small between children in households with unimproved versus improved latrines (1.1\%, 95\% $\mathrm{Cl}$ : 0.4-1.9). There was no statistically significant difference in MAM in children in households practicing open defecation versus those with improved latrines. Children in both households with unimproved latrines and in those practicing open defection had lower mean MUAC than those in households 
TABLE 1

Descriptive characteristics of children with and without moderate acute malnutrition $(N=32,172)$

\begin{tabular}{|c|c|c|c|c|}
\hline & $\begin{array}{l}\text { Severe acute } \\
\text { malnutrition }^{*}\end{array}$ & $\begin{array}{c}\text { Moderate acute } \\
\text { malnutritiont }\end{array}$ & $\begin{array}{c}\text { No moderate } \\
\text { acute }\end{array}$ & $\begin{array}{c}\text { MUAC (median, } \\
\text { IQR) }\end{array}$ \\
\hline Total & 89 & 842 & 31,241 & 14.5 (13.9 to 15.4$)$ \\
\hline \multicolumn{5}{|l|}{ Child's gender§" } \\
\hline Male & $33(37.1 \%)$ & $363(43.1 \%)$ & $15,860(50.8 \%)$ & 14.6 (14 to 15.5$)$ \\
\hline Female & $56(62.9 \%)$ & $479(56.9 \%)$ & $15,381(49.2 \%)$ & 14.5 (13.7 to 15.4$)$ \\
\hline \multicolumn{5}{|l|}{ Age (months) $\S^{\|}$} \\
\hline $6-11$ & $31(34.8 \%)$ & $211(25.1 \%)$ & $2,457(7.9 \%)$ & 13.8 (13 to 14.5$)$ \\
\hline $12-23$ & $33(37.1 \%)$ & $346(41.1 \%)$ & $6,898(22.1 \%)$ & 14 (13.3 to 14.7$)$ \\
\hline $24-35$ & $19(21.4 \%)$ & $188(22.3 \%)$ & $6,961(22.3 \%)$ & $14.5(13.8$ to 15.1$)$ \\
\hline $36-47$ & $5(5.6 \%)$ & $61(7.2 \%)$ & $7,237(23.2 \%)$ & 15 (14.2 to 15.6$)$ \\
\hline $48-59$ & $1(1.1 \%)$ & $36(4.3 \%)$ & 7,688 (24.6\%) & $15.1(14.5$ to 16$)$ \\
\hline \multicolumn{5}{|l|}{ Ethnicity§" } \\
\hline Dafing/Marka & $14(15.7 \%)$ & $146(17.3 \%)$ & $6,818(21.8 \%)$ & $14.6(14$ to 15.5$)$ \\
\hline Bwaba & $16(18.0 \%)$ & $162(19.2 \%)$ & $9,764(31.3 \%)$ & $14.8(14$ to 15.5$)$ \\
\hline Mossi & $3(3.4 \%)$ & $35(4.2 \%)$ & $2,237(7.2 \%)$ & 14.6 (14 to 15.5$)$ \\
\hline Samo & $6(6.7 \%)$ & $26(3.1 \%)$ & $573(1.8 \%)$ & 14.5 (13.6 to 15$)$ \\
\hline Peulh & $28(31.5 \%)$ & 205 (24.5\%) & $4,375(14.0 \%)$ & $14.2(13.5$ to 15$)$ \\
\hline Other & $15(16.7 \%)$ & $185(22.0 \%)$ & $5,067(16.2 \%)$ & $14.3(13.5$ to 15$)$ \\
\hline Missing & $7(7.9 \%)$ & $82(9.7 \%)$ & $2,407(7.7 \%)$ & $14.5(13.8$ to 15.4$)$ \\
\hline \multicolumn{5}{|l|}{ Religion§§" } \\
\hline Muslim & 65 (73.0\%) & 585 (69.5\%) & $18,281(58.5 \%)$ & 14.5 (13.7 to 15.3$)$ \\
\hline Catholic & 15 (16.9\%) & $102(12.1 \%)$ & $7,041(22.5 \%)$ & $14.8(14$ to 15.5$)$ \\
\hline Protestant & $1(1.1 \%)$ & $47(5.6 \%)$ & $2,402(7.7 \%)$ & 14.8 (15 to 15.5$)$ \\
\hline Animist & $1(1.1 \%)$ & $26(3.1 \%)$ & $992(3.2 \%)$ & $14.6(14$ to 15.5$)$ \\
\hline Other & 0 & 0 & $118(0.4 \%)$ & $14.5(14$ to 15.3$)$ \\
\hline Missing & $7(7.9 \%)$ & $82(9.7 \%)$ & $2,407(7.7 \%)$ & 14.5 (13.8 to 15.4$)$ \\
\hline MUAC, median $(I Q R) \S$ & $11(10.5$ to 11$)$ & 12 (12 to 12.2$)$ & $14.6(14$ to 15.5$)$ & N/A \\
\hline \multicolumn{5}{|l|}{ Latrine type $\S^{\|}$} \\
\hline Improved & $6(6.7 \%)$ & $31(3.7 \%)$ & $1,776(5.7 \%)$ & 14.7 (14 to 15.5$)$ \\
\hline Unimproved & $45(50.6 \%)$ & $494(58.7 \%)$ & $16,701(53.5 \%)$ & $14.5(13.8$ to 15.4$)$ \\
\hline Open defecation & $38(42.6 \%)$ & $317(37.7 \%)$ & $12,757(40.8 \%)$ & $14.5(13.9$ to 15.4$)$ \\
\hline \multicolumn{5}{|l|}{ Well type $\|$} \\
\hline Borehole & $15(16.9 \%)$ & 105 (12.5\%) & 4,397 (14.1\%) & $14.7(14$ to 15.5$)$ \\
\hline Shallow dug well & $74(83.2 \%)$ & $737(87.5 \%)$ & $26,837(85.9 \%)$ & 14.5 (13.8 to 15.4$)$ \\
\hline $\begin{array}{l}\text { Number of rooms in } \\
\text { household, median (IQR) }\end{array}$ & 3 (2 to 5$)$ & 3 (2 to 5$)$ & 3 (2 to 4$)$ & N/A \\
\hline \multicolumn{5}{|c|}{ Household owns mobile phone } \\
\hline Yes & $69(78.4 \%)$ & $678(80.9 \%)$ & $25,073(80.8 \%)$ & 14.5 (13.9 to 15.5$)$ \\
\hline No & $19(21.6 \%)$ & $160(19.1 \%)$ & $25,073(19.2 \%)$ & $14.5(13.8$ to 15.4$)$ \\
\hline \multicolumn{5}{|l|}{ Household owns radio§" } \\
\hline Yes & $31(34.8 \%)$ & $391(46.8 \%)$ & $15,067(48.7 \%)$ & 14.5 (13.9 to 15.5$)$ \\
\hline No & $58(65.2 \%)$ & $444(53.2 \%)$ & $15,861(51.3 \%)$ & 14.5 (13.8 to 15.4$)$ \\
\hline Household wealth index $\neq$ & $-0.4(-1.7$ to 0.5$)$ & $-0.2(-1.1$ to 1.1$)$ & $-0.1(-1.1$ to 1.3$)$ & N/A \\
\hline
\end{tabular}

ANOVA = analysis of variance; IQR = interquartile range; $\mathrm{MUAC}=$ mid-upper arm circumference; $\mathrm{N} / \mathrm{A}=$ not applicable.

* Defined as MUAC $<11.5 \mathrm{~cm}$.

$\dagger$ Defined as MUAC $\geq 11.5 \mathrm{~cm}$ to $<12.5 \mathrm{~cm}$.

$\ddagger$ Principal components analysis combining a series of questions related to household resources; responses available for $N=27,480$ children.

$\S P<0.05$ across categories for malnutrition status by Fisher's exact test (categorical variable) or ANOVA (continuous variable).

$\| P<0.05$ for MUAC by ANOVA.

with an improved latrine (Table 3), consistent with approximately $0.15 \mathrm{~cm}(15 \mathrm{~mm})$ reduced MUAC in households without an improved latrine compared with those with an improved latrine. This relationship was attenuated when adjusting for ethnicity, religion, and household wealth index (Table 3).
Household use of a dug well compared with a borehole for drinking water during the dry season was not associated with MAM (Table 2). On average, children in households that primarily used a dug well had $0.10 \mathrm{~cm}(10 \mathrm{~mm})$ smaller MUAC than those that used a borehole (mean difference $-0.10,95 \%$

TABLE 2

Associations between household-level water and sanitation characteristics and MAM (mid-upper arm circumference < 12.5 cm)

\begin{tabular}{|c|c|c|c|c|c|c|c|}
\hline & \multirow[b]{2}{*}{$\%$ MAM } & \multicolumn{2}{|l|}{ Bivariate } & \multicolumn{2}{|c|}{ Multivariable* $^{\star}$} & \multicolumn{2}{|c|}{ Multivariable† } \\
\hline & & OR $(95 \% \mathrm{Cl})$ & $P$-value & aOR $(95 \% \mathrm{Cl})$ & $P$-value & aOR (95\% Cl) & $P$-value \\
\hline \multicolumn{8}{|l|}{ Latrine type } \\
\hline Improved & $37(2.0 \%)$ & 1.00 & - & 1.00 & - & 1.00 & - \\
\hline Unimproved & $539(3.1 \%)$ & 1.55 (1.08 to 2.22$)$ & 0.02 & 1.60 (1.11 to 2.31$)$ & 0.01 & $1.24(0.85$ to 1.81$)$ & 0.28 \\
\hline Open defecation & $355(2.7 \%)$ & $1.34(0.93$ to 1.93$)$ & 0.12 & 1.36 (0.94 to 1.98$)$ & 0.11 & $1.06(0.72$ to 1.57$)$ & 0.76 \\
\hline \multicolumn{8}{|l|}{ Well type } \\
\hline Borehole & $111(2.6 \%)$ & 1.00 & & 1.00 & & 1.00 & \\
\hline Shallow dug well & $811(2.9 \%)$ & $1.13(0.92$ to 1.38$)$ & 0.23 & $1.12(0.91$ to 1.37$)$ & 0.29 & $1.02(0.82$ to 1.25$)$ & 0.89 \\
\hline
\end{tabular}

*Adjusted for child's age, gender, number of rooms in the household, and household's mobile phone and radio ownership $(N=32,172)$.

† One adjusted for child's age, gender, number of rooms in the household, household wealth index, ethnicity, and religion $(N=27,469)$. 
TABLE 3

Associations between household-level water and sanitation characteristics and MUAC in $\mathrm{cm}$

\begin{tabular}{|c|c|c|c|c|c|c|c|}
\hline & \multirow[b]{2}{*}{$\begin{array}{l}\text { MUAC median (interquartile } \\
\text { range) }\end{array}$} & \multicolumn{2}{|l|}{ Bivariate } & \multicolumn{2}{|l|}{ Multivariable $^{*}$} & \multicolumn{2}{|l|}{ Multivariable† } \\
\hline & & Mean difference $(\mathrm{cm})(95 \% \mathrm{Cl})$ & $P$-value & $\begin{array}{l}\text { Adjusted mean difference }(\mathrm{cm}) \\
(95 \% \mathrm{Cl})\end{array}$ & $P$-value & $\begin{array}{l}\text { Adjusted mean difference }(\mathrm{cm}) \\
(95 \% \mathrm{Cl})\end{array}$ & $\begin{array}{c}P- \\
\text { value }\end{array}$ \\
\hline \multicolumn{8}{|l|}{ Latrine type } \\
\hline Improved & 14.7 (14.0 to 15.5$)$ & Ref & - & Ref & - & Ref & - \\
\hline Unimproved & 14.5 (13.8 to 15.4$)$ & $-0.15(-0.21$ to 0.09$)$ & $<0.001$ & $-0.16(-0.21$ to -0.10$)$ & $<0.001$ & $-0.08(-0.14$ to -0.01$)$ & 0.02 \\
\hline $\begin{array}{l}\text { Open } \\
\text { defecation }\end{array}$ & $14.5(13.9$ to 15.4$)$ & $-0.13(-0.19$ to -0.07$)$ & $<0.001$ & $-0.14(-0.20$ to -0.08$)$ & $<0.001$ & $-0.08(-0.15$ to -0.02$)$ & 0.01 \\
\hline \multicolumn{8}{|l|}{ Well type } \\
\hline Borehole & 14.6 (14.0 to 15.5$)$ & Ref & & Ref & & Ref & \\
\hline $\begin{array}{l}\text { Shallow dug } \\
\text { well }\end{array}$ & 14.5 (13.8 to 15.4$)$ & $-0.10(-0.15$ to -0.06$)$ & $<0.001$ & $-0.10(-0.14$ to -0.06$)$ & $<0.001$ & $-0.07(-0.12$ to -0.03$)$ & 0.001 \\
\hline
\end{tabular}

Cl: -0.14 to -0.06 ; Table 3 ), which was similar in models adjusting for ethnicity, religion, and household wealth index. The percentage of households using a dug well during rainy season was similar to that in dry season, and results of the association of drinking water source with nutritional status were similar, regardless of season.

\section{DISCUSSION}

In this large population-based survey of children in northwestern Burkina Faso, we found small, but statistically significant, associations between household latrine type and nutritional status, as determined by MUAC. At the individual level, these differences may not translate to substantial differences in nutritional status for children. An absolute reduction of $1 \%$ of acute malnutrition could represent large differences in absolute numbers of children at the population level. However, small effect sizes are more likely to be due to bias in large observational studies as a smaller amount of bias would be required to account for the association, and this association could be due, at least in part, to unmeasured confounding. In models adjusting for the child's ethnicity, religion, and the full household wealth index, relationships were generally attenuated. This could mean that models not adjusting for these variables had unmeasured confounding or that the population without missing data for these variables is different from that with missing data. Mid-upper arm circumference is the most commonly used indicator for community-based screening for malnutrition and has been shown to be a better predictor of mortality than other anthropometric measurements. ${ }^{10}$ If true, any benefit of improved latrine access could yield important population-level benefits.

Access to an improved latrine and improved water sources is hypothesized to lead to better nutritional outcomes via a reduction in enteric pathogen transmission. Trials of rural sanitation and water quality interventions have shown mixed effects for implementation of WASH interventions on diarrhea prevalence and linear growth. ${ }^{1-4,15-18}$ In areas with high prevalence of diarrhea, it may be difficult to reduce pathogen transmission via WASH and WASH behavior-related interventions. Results of trials may be discordant from our results for several reasons, including confounding by socioeconomic status or other common causes of household WASH access and child nutritional status. For example, households with greater socioeconomic resources likely feed their children more diverse and nutrient-rich diets, which improves nutritional status, ${ }^{19}$ and may also have better access to improved sanitation. Although models were adjusted for some proxies of socioeconomic status, including radio and mobile phone ownership, there is possibly residual confounding by socioeconomic status that we were unable to account for. ${ }^{6}$ This is partially supported by attenuation of effects in models that were adjusted for a household wealth index that combined responses for 20 different household use items.

Drinking water sources were categorized as borehole versus dug well in this survey. We did not ask specifically about access to improved drinking water sources nor did we ask about handwashing availability or drinking water storage. Boreholes are deeper than the shallower dug wells and may be less vulnerable to contamination from other environmental sources; however, both have been shown to harbor Escherichia coli. ${ }^{20}$ A lack of difference in acute malnutrition by type of well may be explained by the presence of potentially pathogenic bacteria present in both drinking water sources. However, we did not collect water samples from water sources in the study area and cannot comment on the presence of pathogenic bacteria in this region.

Mid-upper arm circumference is an easy-to-use measure for identifying children with MAM and SAM and is commonly used for community-based screening. ${ }^{21}$ Acute malnutrition can also be defined based on the WHZ. Mid-upper arm circumference has been shown to be a better predictor of mortality than the WHZ in a similar population-based setting. ${ }^{10}$ Clinic-based settings have demonstrated that these two methods identify different subgroups of children and, in some cases, have shown that children with SAM diagnosed via WHZs have higher mortality than those with MUAC. ${ }^{22,23}$ Mid-upper arm circumference screening alone may miss some children with MAM or SAM. The current study was part of a large, community-based trial, ${ }^{13}$ and $\mathrm{WHZ}$ measurement was not feasible at this operational scale. However, the relationship between access to WASH and nutritional status may differ based on different anthropometric indicators.

In addition to issues outlined earlier, there are several limitations of this analysis to note. Although more than 30,000 children were included in this analysis, the number of children with MAM and in households with improved latrines was limited, which means that there was limited power for some 
comparisons. As previously mentioned, we did not have anthropometric indicators, other than MUAC, and cannot comment on associations between WASH indicators and chronic malnutrition. We did not measure diarrhea or measurements of pathogen burden to understand whether households with improved WASH had lower infection burden because the primary goal of the initial household survey was to understand basic household resources and characteristics. Household WASH indicators were limited to toilet type used by the household and source of drinking water. Because of resource limitations in the study, we did not conduct formal structured observations or collect additional information about WASH variables that may be important, such as drinking water storage or handwashing facilities. Finally, household characteristics were measured by the head of household interview and not objectively verified. Heads of households may have underreported open defecation because of social desirability bias. We do not anticipate that this misclassification would be differential with respect to MUAC measurements and thus would most likely bias effect measures toward the null. For example, we found that unimproved latrine usage may be associated with MAM compared with improved latrine usage, but not open defecation. If open defecation was underreported because of social desirability bias, this could potentially explain this result. Strengths of this study include the large sample size that is representative of the entire district and the use of longitudinal data. The longitudinal data available in this study decrease some of the potential for reverse causality that would be introduced by the use of crosssectional data.

In this large census-based study of children in northwestern Burkina Faso, we found a small, but statistically significant, association between access to improved latrines and borehole versus dug wells and MUAC in children younger than 5 years. Children in households with improved access to WASH may have reduced enteric pathogen exposure that improves their nutritional status; however, this relationship may also be due to residual confounding by socioeconomic status. Collection of data related to diarrhea and environmental samples could improve estimates and understanding mechanisms of the effect of latrine conditions and different water sources on pathogen exposure, diarrhea, and nutritional status.

Received May 21, 2020. Accepted for publication October 16, 2020. Published online February 8, 2021.

Financial support: The CHAT study was supported by the Bill \& Melinda Gates Foundation (OPP1187628).

Disclosure: The study was reviewed and approved by the Comite National d'Ethique pour la Recherche (National Ethics Committee of Burkina Faso) in Ouagadougou, Burkina Faso, and the Institutional Review Board the University of California, San Francisco, in the United States. Written informed consent was obtained from each head of households for participation in the household survey and census and from each child's caregiver for participation in the study.

Authors' addresses: Mamadou Bountogo, Mamadou Ouattara, Ali Sié, Guillaume Compaoré, Clarisse Dah, Valentin Boudo, and Alphonse Zakane, Centre de Recherche en Sante de Nouna, Nouna, Burkina Faso, E-mails: drbountogo@yahoo.fr, md.ouattara@yahoo.fr, sieali@yahoo.fr, gcompaore2@gmail.com, n.clarissedah@yahoo.fr, valentinboudo@gmail.com, and al_zakane@yahoo.fr. Elodie Lebas, Jessica M. Brogdon, William W. Godwin, Ying Lin, Benjamin F. Arnold, and Catherine E. Oldenburg, Francis I, Proctor Foundation, University of California, San Francisco, CA, E-mails: elodie.lebas@ucsf.edu, jessica.brogdon@ucsf.edu, william.godwin@ucsf.edu, ying.lin@ucsf. edu, ben.arnold@ucsf.edu, and catherine.oldenburg@ucsf.edu.

This is an open-access article distributed under the terms of the Creative Commons Attribution (CC-BY) License, which permits unrestricted use, distribution, and reproduction in any medium, provided the original author and source are credited.

\section{REFERENCES}

1. Pickering AJ et al., 2019. The WASH Benefits and SHINE trials: interpretation of WASH intervention effects on linear growth and diarrhoea. Lancet Glob Health 7: e1139-e1146.

2. Luby SP et al., 2018. Effects of water quality, sanitation, handwashing, and nutritional interventions on diarrhoea and child growth in rural Bangladesh: a cluster randomised controlled trial. Lancet Glob Health 6: e302-e315.

3. Null $C$ et al., 2018. Effects of water quality, sanitation, handwashing, and nutritional interventions on diarrhoea and child growth in rural Kenya: a cluster-randomised controlled trial. Lancet Glob Heal 6: e316-e329.

4. Humphrey JH et al., 2019. Independent and combined effects of improved water, sanitation, and hygiene, and improved complementary feeding, on child stunting and anaemia in rural Zimbabwe: a cluster-randomised trial. Lancet Glob Heal 7: e132-e147.

5. Checkley W, Gilman RH, Black RE, Epstein LD, Cabrera L, Sterling $\mathrm{CR}, 2004$. Effect of water and sanitation on childhood health in a poor Peruvian peri-urban community. Lancet 363: 112-118.

6. Arnold BF, Null C, Luby SP, Colford JM, 2018. Implications of WASH benefits trials for water and sanitation. Lancet Glob Heal 6: e616-e617.

7. Tickell KD, Sharmin R, Deichsel EL, Lamberti LM, Walson JL, Faruque ASG, Pavlinac PB, Kotloff KL, Chisti MJ, 2020. The effect of acute malnutrition on enteric pathogens, moderate-tosevere diarrhoea, and associated mortality in the Global Enteric Multicenter Study cohort: a post-hoc analysis. Lancet Glob Heal 8: e215-e224.

8. Guerrant RL, Schorling JB, McAuliffe JF, Auxiliadora de Souza M, 1992. Diarrhea as a cause and an effect of malnutrition: diarrhea prevents catch-up growth and malnutrition increases diarrhea frequency and duration. Am J Trop Med Hyg 47: 28-35.

9. Mata L, 1992. Diarrheal disease as a cause of malnutrition. $A m \mathrm{~J}$ Trop Med Hyg 47: 16-27.

10. O'Brien KS et al., 2020. Comparison of anthropometric indicators to predict mortality in a population-based study of children under 5 in Niger. Public Health Nutr 23: 538-543.

11. Grellety E, Krause LK, Eldin MS, Porten K, Isanaka S, 2015. Comparison of weight-for-height and mid-upper arm circumference (MUAC) in a therapeutic feeding programme in South Sudan: is MUAC alone a sufficient criterion for admission of children at high risk of mortality? Public Health Nutr 18: 2575-2581.

12. Sie A, Louis VR, Gbangou A, Müller O, Niamba L, Stieglbauer G, Yé M, Kouyaté B, Sauerborn R, Becher H, 2010. The health and demographic surveillance system (HDSS) in Nouna, Burkina Faso, 1993-2007. Glob Health Action 3: 5284.

13. Sié $A$ et al., 2019. A double-masked placebo-controlled trial of azithromycin to prevent child mortality in Burkina Faso, West Africa: Community Health with Azithromycin Trial (CHAT) study protocol. Trials 20: 675

14. Sié A et al., 2020. Insecticide-treated bed net access and use among preschool children in Nouna district, Burkina Faso. Int Health 12: 164-169.

15. Clasen T, Alexander K, Sinclair D, Boisson S, Peletz R, Chang HH, Majorin F, Cairncross S, 2015. Interventions to improve water quality for preventing diarrhoea. Cochrane Database Syst Rev 10: CD004794.

16. Wolf $\mathrm{J}$ et al., 2014. Assessing the impact of drinking water and sanitation on diarrhoeal disease in low- and middle-income settings: systematic review and meta-regression. Trop Med Int Health 19: 928-942.

17. Pickering AJ, Djebbari H, Lopez C, Coulibaly M, Alzua ML, 2015. Effect of a community-led sanitation intervention on child 
diarrhoea and child growth in rural Mali: a cluster-randomised controlled trial. Lancet Glob Health 3: e701-e711.

18. Cumming $O$ et al., 2019. The implications of three major new trials for the effect of water, sanitation and hygiene on childhood diarrhea and stunting: a consensus statement. BMC Med 17: 173.

19. Sie A et al., 2018. Dietary diversity and nutritional status among children in rural Burkina Faso. Int Health 382: 426-427.

20. Maponga BA, Chirundu D, Gombe NT, Tshimanga M, Shambira G, Takundwa L, 2013. Risk factors for contracting watery diarrhoea in Kadoma city, Zimbabwe, 2011: a case control study. BMC Infect Dis 13: 567.
21. Alé FGB et al., 2016. Mothers screening for malnutrition by midupper arm circumference is non- inferior to community health workers: results from a large-scale pragmatic trial in rural Niger. Arch Public Health 74: 38.

22. Grellety E, Golden MH, 2018. Severely malnourished children with a low weight-for-height have a higher mortality than those with a low mid-upper-arm- circumference: I. Empirical data demonstrates Simpson's paradox. Nutr J 17: 79.

23. Laillou A, Prak S, de Groot R, Whitney S, Conkle J, Horton L, Un SO, Dijkhuizen MA, Wieringa FT, 2014. Optimal screening of children with acute malnutrition requires a change in current WHO guidelines as MUAC and WHZ identify different patient groups. PLoS One 9: e101159. 